\title{
Exchange effect and magneto-plasmon mode dispersion in an anisotropic two-dimensional electronic system
}

\author{
Xiaoguang $\mathrm{Wu}$ \\ SKLSM, Institute of Semiconductors, Chinese Academy of Sciences, Beijing 100083, China
}

\begin{abstract}
The exchange effect and the magneto-plasmon mode dispersion are studied theoretically for an anisotropic two-dimensional electronic system in the presence of an uniform perpendicular magnetic field. Employing an effective low-energy model with anisotropic effective masses, which is relevant for a monolayer of phosphorus, the exchange effect due to the electron-electron interaction is treated within the self-consistent Hartree-Fock approximation. The magneto-plasmon mode dispersion is obtained by solving a Bethe-Salpeter equation for the electron density-density correlation function within the ladder diagram approximation. It is found that the exchange effect is reduced in the anisotropic system in comparison with the isotropic one. The magneto-plasmon mode dispersion shows a clear dependence on the direction of the wave vector.

PACS numbers: 78.20.Ls, 73.21.-b, 73.22.Lp, 81.05.Zx
\end{abstract}

\section{INTRODUCTION}

In recent years, the black phosphorus has attracted great attention because of its interesting physical properties and its great potential in the electronic and electrooptical device applications,$\frac{1,2}{2}$

Many experimental works are devoted to the material growth, the physical property characterization, and the device exploration $\underline{\underline{1}}^{-\underline{6}}$ There is also a considerable amount of theoretical investigations that concerning the electronic band structures, $\stackrel{7-9}{-}$ the Landau levels and the anisotropic optical properties, $\stackrel{10}{\underline{1}-12}$ the plasmon at zero magnetic field, $\stackrel{13-16}{=}$ the topological and edge states, $\stackrel{17,18}{=}$ the anisotropic composite fermions, $\stackrel{19,20}{,}$, the electron substrate phonon coupling, $\stackrel{21}{=}$ and the tuning of the band gap by a bias electric field $\stackrel{22}{=}$ It is interesting to note that the monolayer black phosphorus may provide an alternative two-dimensional electronic system to study the influence of interplay between the anisotropy and the electronelectron interaction. This interplay has been studied in a GaAs quantum well recently ${ }^{23}$ In these theoretical investigations, we feel that the many-body effects arising from the electron-electron interaction is less extensively studied. More studies on the many-particle effect is desired.

This motivates the present study. In this paper, we will study the exchange effect in an anisotropic twodimensional electronic system, in the presence of a perpendicular magnetic field, due to the electron-electron interaction within the self-consistent Hartree-Fock approximation. This exchange effect is an important ingredient for the explanation of the quantum oscillation displayed in the magneto-transport property of an isotropic two-dimensional electronic system. For an anisotropic system, one expects that it is important as well. We will also study the magneto-plasmon mode dispersion beyond the random-phase-approximation. The magnetoplasmon was observed experimentally for the isotropic two-dimensional electronic system. 24

The present paper is organized as follows: in the sec- tion II, the approach used is briefly presented. In the section III, our theoretical results are shown and discussed. Finally, a brief summary is provided in the last section.

\section{FORMULATIONS AND CALCULATIONS}

In this paper, the anisotropic two-dimensional (2D) electronic system in the $x y$ plane is modelled by the following Hamiltonian

$H=\frac{1}{2 m_{1}}\left(p_{x}+\frac{e A_{x}}{c}\right)^{2}+\frac{1}{2 m_{2}}\left(p_{y}+\frac{e A_{y}}{c}\right)^{2}+g^{*} \mu_{B} B s_{z}$,

in the presence of an uniform perpendicular magnetic field $B$. This effective low-energy Hamiltonian can be used to describe the conduction band of a monolayer of phosphorus. $: 11$ The valence band is omitted for simplicity and clarity. The $2 \mathrm{D}$ electronic system displays an anisotropy via two different effective masses $m_{1}$ and $m_{2}$. $g^{*}$ is the effective $g$-factor, and $s_{z}$ is the electron spin operator. The electron-electron interaction is assumed to be isotropic and the Coulomb potential is a function of the distance between two electrons.

By choosing a gauge as $\mathbf{A}=(0, B x, 0)$, one obtains the single-particle energy level $(\sigma= \pm 1)$

$$
\varepsilon_{l, \sigma}^{0}=\hbar \omega_{c}(l+1 / 2)+g^{*} \mu_{B} B \sigma / 2,
$$

with $\omega_{c}=e B /\left(c \sqrt{m_{1} m_{2}}\right)$. The Landau levels are degenerated, and this degeneracy leads to an area density $n_{B}=e B /(2 \pi \hbar c)=1 /\left(2 \pi l_{B}^{2}\right)$, independent of the effective mass parameters. The corresponding one-particle wave function is given by

$$
\phi_{l, k_{y}}(\mathbf{r})=\left[e^{i k_{y} y} / \sqrt{L_{y}}\right]\left[\sqrt{\alpha} \psi_{l}\left(\alpha\left(x-x_{0}\right)\right)\right],
$$

with $x_{0}=l_{B}^{2} k_{y}, \alpha=\left(m_{1} \omega_{c} / \hbar\right)^{1 / 2}$, and

$$
\psi_{n}(x)=\left[\pi^{1 / 2} 2^{n} n !\right]^{-1 / 2} e^{-x^{2} / 2} H_{n}(x),
$$

with $H_{n}(x)$ the Hermit polynomial ${ }^{25}$ Note that the length scale $\alpha$ depends on the effective mass parameter. 
It is interesting to note that the well-known Kohn's theorem is also valid for this anisotropic $2 \mathrm{D}$ electronic system. ${ }^{26}$ This leads to a constrain on the magnetoplasmon mode dispersion at small wave vectors.

The exchange effect due to the electron-electron interaction is treated within the self-consistent Hartree-Fock approximation. ${ }^{27,28}$ The imaginary time Green's function can be written as

$$
G_{\sigma}^{0}\left(\mathbf{r}, \mathbf{r}^{\prime}, i \omega_{n}\right)=\sum_{l, k_{y}} \frac{\phi_{l, k_{y}}(\mathbf{r}) \phi_{l, k_{y}}^{*}\left(\mathbf{r}^{\prime}\right)}{i \omega_{n}-\left(\varepsilon_{l, \sigma}^{0}-\mu\right) / \hbar}
$$

for the non-interacting system. ${ }^{27}$ After taking into account the exchange effect, the Green's function becomes

$$
G_{\sigma}\left(\mathbf{r}, \mathbf{r}^{\prime}, i \omega_{n}\right)=\sum_{\lambda, k_{y}} \frac{\psi_{\lambda, k_{y}}(\mathbf{r}) \psi_{\lambda, k_{y}}^{*}\left(\mathbf{r}^{\prime}\right)}{i \omega_{n}-\left(\varepsilon_{\lambda, \sigma}-\mu\right) / \hbar}
$$

with $\psi_{\lambda, \sigma}(\mathbf{r})=\sum_{m} C_{\lambda, \sigma ; m} \phi_{m, k_{y}}(\mathbf{r})$. The Landau level energy $\varepsilon_{\lambda, \sigma}$, with the exchange effect included, is still independent of $k_{y}$. The expansion coefficients $C_{\lambda, \sigma ; m}$ needs to be calculated self-consistently.

The magneto-plasmon mode dispersion is obtained by solving a Bethe-Salpeter equation for the electron density-density correlation function within the ladder diagram approximation. ${ }^{27}$ The electron density-density correlation function can be written as

$$
D\left(\mathbf{q}, i \omega_{n}\right)=\sum_{\lambda} \frac{\pi_{\lambda}(\mathbf{q})}{i \omega_{n}-\omega_{\lambda}(\mathbf{q})}
$$

where $\omega_{\lambda}(\mathbf{q})$ gives the magneto-plasmon mode dispersion, and $\pi_{\lambda}(\mathbf{q})$ gives the corresponding oscillator strength. The damping of magneto-plasmon can not be obtained in this approach. For an isotropic system, the magneto-plasmon mode dispersion was calculated previously. ${ }^{29.30}$ The effect of disorder was studied. ${ }^{31}$ The influence of multiple subbands and spin-orbit coupling was also considered previously for the isotropic system. 32

There is a simple scaling relation when the electron density-density correlation function is calculated for an non-interaction 2D electronic system. By denoting the electron density-density correlation function of an noninteracting isotropic 2D system, in the presence of a perpendicular magnetic field, as $D_{\text {iso }}^{0}\left(q l_{B}, \omega / \omega_{c}\right)$, then the corresponding electron density-density correlation function for the anisotropic case can be obtained as

$$
D_{\text {aniso }}^{0}\left(\mathbf{q} l_{B}, \omega / \omega_{c}\right)=D_{\text {iso }}^{0}\left(p l_{B}, \omega / \omega_{c}\right),
$$

with $p_{x}=q_{x}\left(m_{2} / m_{1}\right)^{1 / 2}$ and $p_{y}=q_{y}\left(m_{1} / m_{2}\right)^{1 / 2}$. This scaling relation can be easily verified by directly calculating the one-bubble electron density-density correlation function. ${ }^{27}$ With the help of this scaling relation, a qualitative picture can be quickly drawn for the magneto-plasmon mode dispersion within the randomphase-approximation from previously known theoretical result.

\section{RESULTS AND DISCUSSIONS}

In this paper, we will use $\hbar \omega_{c}$ as the energy scale, $l_{B}$ as the length scale. The wave vector will be scaled by $1 / l_{B}$. The electron density enters our calculation via the filling factor $\nu=n_{e} / n_{B}$ for a single spin. As the Landau level energy will be scaled by $\hbar \omega_{c}$, and the magneto-plasmon mode frequency will be scaled by $\omega_{c}$, only the ratio of two effective masses $m_{1}$ and $m_{2}$ is needed in our calculation. However, the values of $m_{1}$ and $m_{2}$ used in the calculation for a particular figure will be given individually without causing any confusion.

For clarity, we will take the effective $g$-factor a vanishingly small value. However, the occupation of the Landau levels is assumed to be spin resolved. The spin-down Landau level is assumed to be occupied first, and the spin-up Landau level to be occupied next. This approximation scheme has to be modified when one considers the case of a tilted and large magnetic field that the Zeeman spin splitting may become larger than the separation of two neighboring Landau levels. The system temperature is assumed to be zero.

Another parameter enters our calculation is the strength of the electron-electron interaction. This parameter is introduced via the following dimensionless quantity $v_{e}=\sqrt{2} e^{2} /\left(\epsilon_{s} l_{B}\right) /\left(\hbar \omega_{c}\right)$, with $\epsilon_{s}$ the background dielectric constant. We takes $v_{e}<1$ in accordance with the nature of the perturbation theory employed in the present work.

In Fig.1, the energy of a few low Landau levels versus the filling factor is plotted. For an even filling factor of a value $2 n, n$ spin-down Landau levels and $n$ spinup Landau levels are occupied. This leads to the same exchange correction to the spin-down Landau level and the spin-up Landau level. In this case, the spin splitting of the Landau level will be purely come from the Zeeman spin splitting. As we choose $g^{*}=0$, there is no spin splitting of the Landau level shown in the figure, as one expects.

For an odd filling factor of a value $2 n+1$, there are $n+1$ spin-down Landau levels occupied, and $n$ spin-up Landau levels occupied. The $(n+1)$-th spin-up Landau is empty. This results in a larger energy shift, due to the exchange effect, for the spin-down Landau level. Consequently, a spin-splitting of the Landau level occurs.

When the filling factor takes a fractional value, an integer number of Landau levels for one spin are fully occupied, while for the other spin, one Landau level will be partially occupied. Thus, one observes that the spindown (spin-up) Landau level energy remains constant, and the spin-up (spin-down) Landau level energy decreases linearly as the filling factor increases from one integer to the next higher integer. This picture is qualitative the same as in the case of an isotropic electronic system. ${ }^{29-32}$ In the Fig.1, the parameters used are $m_{1}=1, m_{2}=2.4$, and $v_{e}=0.61$. Note that only the ratio $m_{1} / m_{2}$ matters as mentioned previously. In a monolayer of phosphorus, the value of $m_{1} / m_{2}$ can be quite 
large,,$\frac{1}{,}$ we have chosen a moderate value instead.

In Fig.2, the energy of the lowest two Landau levels is shown versus the effective mass ratio $m_{2} / m_{1}$. There is an symmetry. The energy levels will be the same when $m_{2} / m_{1}=r$ and when $m_{2} / m_{1}=1 / r$. This reflects the fact that the physics must not change when one denotes the direction with the effective mass $m_{1}$ as the $x$-axis or as the $y$-axis. One observes that the correction to the Landau levels due to the exchange effect is largest when $m_{2} / m_{1}=1$. This is because that when $m_{2} / m_{1} \neq 1$, the $2 \mathrm{D}$ electronic system has a lower symmetry, the exchange effect leads to a mixing of Landau levels, and this mixing effect reduces the energy shift due to the exchange interaction. This can be viewed as the reduction of the exchange effect due to the anisotropy.

One also observes that, the depth of two smile curves shown in the Fig.2 is different. This shows that the spin splitting of a Landau level is also reduced due the anisotropy in a 2D electronic system. The above features shown for the lowest two Landau levels also exist for the higher Landau levels. In the Fig.2, the parameters used are filling factor $\nu=3.1$ and $v_{e}=0.71$.

Next, let us examine the magneto-plasmon mode dispersion. In Fig.3, the frequency of magneto-plasmon modes around $\omega_{c}$ versus the amplitude of the wave vector is plotted for three different integer filling factors, $\nu=1,2,3$. The other parameters used are $v_{e}=0.87$, $m_{1}=1$, and $m_{2}=3$. The direction of the wave vector is characterized by an angle $\varphi\left(q_{x}=q \cos (\varphi)\right)$, and it takes a fixed value of $\varphi=\pi / 6$ for the dispersion curves shown in the Fig.3. The magneto-plasmon modes are determined by the poles in the electron density-density correlation function, 27,28 There are many modes, but in this paper, we will focus on the mode whose frequency is around $\omega_{c}$.

In the case of $\nu=1$, only the $n=0$ spin-down Landau level is occupied, the electron density excitation around $\omega_{c}$ should arise from a transition between the $n=0$ spindown Landau level and the $n=1$ spin-down Landau level. As the spin-up Landau levels are empty, there is no spin-up Landau level transition. Therefore, one would expect only one magneto-plasmon mode around $\omega / \omega_{c}=1$. In the cases of $\nu=2$ and $\nu=3$, following a similar argument, one would expect two magnetoplasmon modes. Our calculation corroborates this hand waving picture.

Note that there is no spin-density excitation shown, as the 2D electronic system studied here has no spin-orbit interaction, and we are limited ourselves to the electron density excitations. The spin-density excitation can be evaluated by summing up a series of slightly different ladder diagrams $\frac{27.28}{2}$ When the filling factor is not an integer, the spin-density excitation will show up as a collective excitation frequency less than $\omega_{c}$, around the Zeeman spin splitting.

The magneto-plasmon modes can be classified into $E_{1}$ modes and $E_{2}$ modes for small wave vectors. They are indicated explicitly in the Fig.3. For small wave vectors, the amplitude of $E_{1}$ modes behaves like $\pi_{\lambda}(\mathbf{q}) \sim q^{2}$. The amplitude of $E_{2}$ modes is much smaller. In the long wave length limit, the shown $E_{1}$ modes dominate, and the corresponding magneto-plasmon mode frequencies approach $\omega_{c}$ as required by the Kohn's theorem ${ }^{26}$

For an isotropic 2D electronic system, the magnetoplasmon mode was observed experimentally by depositing a grating coupler to a surface close to the $2 \mathrm{D}$ electronic system $\stackrel{24}{2}$ However, the wave vector achieved at that time was small. We wish that this theoretical work will inspire more experimental investigations taking the advantage of currently available more advanced chip technology.

The dispersions shown in the Fig.3 display a similar $q$-dependence as that for an isotropic 2D electronic system. ${ }^{29}$ However, for an isotropic system, the magnetoplasmon mode frequency only depends on the magnitude of the wave vector, and is independent of the direction of the wave vector. This is no longer the case for the anisotropic $2 \mathrm{D}$ electronic system. This will be examined next.

In Fig.4, the frequency of magneto-plasmon modes around $\omega_{c}$ is depicted versus the direction of the wave vector, characterized by $\varphi$ the angle between the wave vector $\mathbf{q}$ and the $x$ axis, for the integer filling factors $\nu=1,2,3$. The magnitude of the wave vector is fixed at $q l_{B}=0.75$. The other parameters used are $v_{e}=0.87$, $m_{1}=1$, and $m_{2}=3$. One observes that the magnetoplasmon mode dispersion shows a clear angular dependence. As $\varphi$ changes, the magneto-plasmon mode frequency displays an oscillatory behavior. The amplitude of this oscillation depends on the magnitude of the wave vector, but in a non-monotonic way.

Calculations are carried out for some $q$ values. It is found that $E_{1}$ modes have a larger $\varphi$ oscillations amplitude, and the amplitude for the $E_{2}$ modes is smaller. In the Fig.4, one observes that, the $E_{1}$ magneto-plasmon mode frequency takes its minimal value around $\varphi=\pi / 2$ and $\varphi=3 \pi / 2$. This behavior is only found for some small $q$ values. For some larger $q$ values, the minimal is located around $\varphi=0$, or falls between $\varphi=0$ and $\varphi=\pi / 2$.

It should be pointed out that, the Landau level energy will not change if one exchanges the values of two effective masses $m_{1}$ and $m_{2}$. However, the magneto-plasmon mode dispersions shown in the Fig.3 and Fig.4, does change if one swaps the values of $m_{1}$ and $m_{2}$. This is expected, as the wave function expansion in the Green's function is different for different effective masses.

In Fig.5, we plot the frequency of magneto-plasmon modes versus the amplitude of the wave vector, for a non-integer filling factor $\nu=2.3$. The other parameters used are $v_{e}=0.87, m_{1}=1, m_{2}=3$, and $\varphi=\pi / 6$. For $\nu=2.3$, the occupation of the Landau levels are as follows: $n=0$ spin-down and $n=0$ spin-up Landau levels are fully occupied, $n=1$ spin-down Landau level is partially occupied, and all other Landau levels are empty. The electron density excitations around $\omega_{c}$ could arise (1) from the transition between the $n=0$ to $n=1$ spin- 
down Landau levels, (2) from the transition between the $n=0$ to $n=1$ spin-up Landau levels, and (3) from the transition between the $n=1$ to $n=2$ spin-down Landau levels. One expects 3 modes. In the Fig.5, one observes $E_{1}$ and $E_{2}$ modes as one observed in the Fig.3 for $\nu=2,3$. A new mode appears and is labelled as $E_{3}$. This mode is absent in the Fig.3. This mode arises from the third transition discussed above. We find that, at long wave length (small $q$ ), the dominate mode is still $E_{1}$, the strength of $E_{2}$ and $E_{3}$ modes are much smaller. This is expected from the Kohn's theorem. ${ }^{26}$

In Fig.6, the frequency of magneto-plasmon modes versus the direction of the wave vector is plotted, for the non-integer filling factor $\nu=2.3$, at $q l_{B}=0.75$. Other parameters used are the same as that in the Fig.5, $v_{e}=0.87, m_{1}=1$, and $m_{2}=3$. One observes a clear angular dependence. The magneto-plasmon mode dispersion shows an oscillatory behavior versus $\varphi$. The amplitude of this oscillation is larger for the $E_{1}$ mode, and smaller for the $E_{2}$ mode and $E_{3}$ mode. We find that, this behavior also holds for some other $q$ values.

The band structure of a monolayer of phosphorus is more complicated than the simple anisotropic model used in the present study, $1,2,7,22$ However, we believe that the picture shown here for the magneto-plasmon mode will qualitatively remain when the influence of a more realistic band structure is taken into account. On the other hand, the influence of disorder, of spin-orbit interaction, and of electron-phonon coupling should be studied in the future.

\section{SUMMARY}

In summary, we have used an effective low-energy model with two effective masses to investigate the influence of anisotropy in an interacting two-dimensional electronic system, relevant for a monolayer of phosphorus. The electron-electron interaction induced exchange effect and the magneto-plasmon mode dispersion are studied theoretically. The correction due to the exchange interaction to the Landau levels is evaluated within the self-consistent Hartree-Fock approximation. The magneto-plasmon mode dispersion is calculated by solving a Bethe-Salpeter equation for the electron density-density correlation function within the ladder diagram approximation, beyond the random phase approximation. It is found that the exchange effect is reduced in the anisotropic system in comparison with the isotropic one. The magneto-plasmon mode dispersion shows a strong dependence on the direction of the wave vector.

\section{Acknowledgments}

This work was partly supported NSF of China via projects 61076092 and 61290303 .
1 Xi Ling, Han Wang, Shengxi Huang, Fengnian Xia, and M. S. Dresselhaus, PNAS 112, 4523 (2015).

2 Sheneve Z. Butler, Shawna M. Hollen, Linyou Cao, Yi Cui, Jay A. Gupta, Humberto R. Gutierrez, Tony F. Heinz, Seung Sae Hong, Jiaxing Huang, Ariel F. Ismach, Ezekiel Johnston-Halperin, Masaru Kuno, Vladimir V. Plashnitsa, Richard D. Robinson, Rodney S. Ruoff, Sayeef Salahuddin, Jie Shan, Li Shi, Michael G. Spencer, Mauricio Terrones, Wolfgang Windl, and Joshua E. Goldberger, ACS Nano 7, 2898 (2013).

3 Ryan J. Suess, Mohammad M. Jadidi, Thomas E. Murphy, and Martin Mittendorff, Appl. Phys. Lett. 107, 081103 (2015).

4 Hongtao Yuan, Xiaoge Liu, Farzaneh Afshinmanesh, Wei Li, Gang Xu, Jie Sun, Biao Lian, Alberto G. Curto, Guojun Ye, Yasuyuki Hikita, Zhixun Shen, Shou-Cheng Zhang, Xianhui Chen, Mark Brongersma, Harold Y. Hwang, and Yi Cui, Nat. Nanotechnol. 10, 707 (2015).

${ }^{5}$ Likai Li, Fangyuan Yang, Guo Jun Ye, Zuocheng Zhang, Zengwei Zhu, Wenkai Lou, Xiaoying Zhou, Liang Li, Kenji Watanabe, Takashi Taniguchi, Kai Chang, YayuWang, Xian Hui Chen, and Yuanbo Zhang, Nat. Nanotechnol. 11, 593 (2016).

${ }^{6}$ Nathaniel Gillgren, Darshana Wickramaratne, Yanmeng Shi, Tim Espiritu, Jiawei Yang, Jin Hu, Jiang Wei, Xue Liu, Zhiqiang Mao, Kenji Watanabe, Takashi Taniguchi, Marc Bockrath, Yafis Barlas, Roger K Lake, and Chun Ning Lau, 2D Mater. 2, 011001 (2015).
7 A. N. Rudenko and M. I. Katsnelson, Phys. Rev. B 89, 201408(R) (2014).

8 Pengke Li and Ian Appelbaum, Phys. Rev. B 90, 115439 (2014).

9 J. M. Pereira Jr., and M. I. Katsnelson, Phys. Rev. B 92, 075437 (2015).

10 Tony Low, A. S. Rodin, A. Carvalho, Yongjin Jiang, Han Wang, Fengnian Xia, and A. H. Castro Neto, Phys. Rev. B 90, 075434 (2014).

11 M. Tahir, P. Vasilopoulos, and F. M. Peeters, Phys. Rev. B 92, 045420 (2015).

12 X. Y. Zhou, R. Zhang, J. P. Sun, Y. L. Zou, D. Zhang, W. K. Lou, F. Cheng, G. H. Zhou, F. Zhai, and Kai Chang, Sci. Rep. 5, 12295 (2015).

13 Fengping Jin, Rafael Roldan, Mikhail I. Katsnelson, and Shengjun Yuan, Phys. Rev. B 92, 115440 (2015)

14 P. K. Pyatkovskiy and Tapash Chakraborty, Phys. Rev. B 93, 085145 (2016).

15 Tony Low, Rafael Roldan, Han Wang, Fengnian Xia, Phaedon Avouris, Luis Martin Moreno, and Francisco Guinea, Phys. Rev. Lett. 113, 106802 (2014).

16 Andrei Nemilentsau, Tony Low, and George Hanson, Phys. Rev. Lett. 116, 066804 (2016).

17 Ruixiang Fei, Vy Tran, and Li Yang, Phys. Rev. B 91, 195319 (2015).

18 B. Ostahie and A. Aldea, Phys. Rev. B 93, 075408 (2016).

19 Areg Ghazaryan and Tapash Chakraborty, Phys. Rev. B 92, 165409 (2015). 
20 Ajit C. Balram and J. K. Jain, Phys. Rev. B 93, 075121 (2016).

21 A. Mogulkoc, Y. Mogulkoc, A. N. Rudenko, and M. I. Katsnelson, Phys. Rev. B 93, 085417 (2016).

22 Shengjun Yuan, Edo van Veen, Mikhail I. Katsnelson, and Rafael Roldan, Phys. Rev. B 93, 245433 (2016).

23 M. A. Mueed, D. Kamburov, S. Hasdemir, L. N. Pfeiffer, K. W. West, K. W. Baldwin, and M. Shayegan, Phys. Rev. B 93, 195436 (2016).

24 E, Batke, D. Heitmann, and C.W. Tu, Phys. Rev. B 34, 69511986.

25 E. Merzbacher, Quantum Mechanics (Wiley, New York, 1970).

26 W. Kohn, Phys. Rev. 123, 1242 (1961).

27 A. L. Fetter and J. D. Walecka, Quantum Theory of ManyParticle Systems (McGraw-Hill, New York, 1971).

28 G. D. Mahan, Many-Particle Physics (Plenum, New York, 1990).

29 C. Kallin and B. I. Halperin, Phys. Rev. B 30, 5655 (1984).

30 A. H. MacDonald, J. Phys. C 18, 1003 (1985).

31 D. Antoniou and A. H. MacDonald, Phys. Rev. B 46, 15225 (1992).

${ }^{32}$ X. G. Wu, L. J. Xu, and H. Z. Zheng, Phys. Rev. B 74,
165309 (2006).

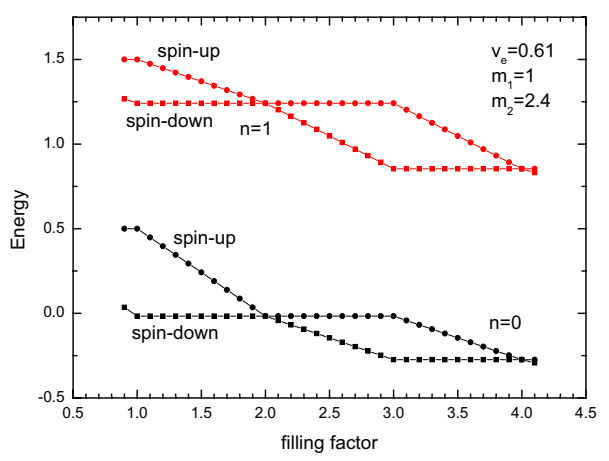

FIG. 1: (Color online) The energy of a few low-lying Landau levels versus the filling factor. 


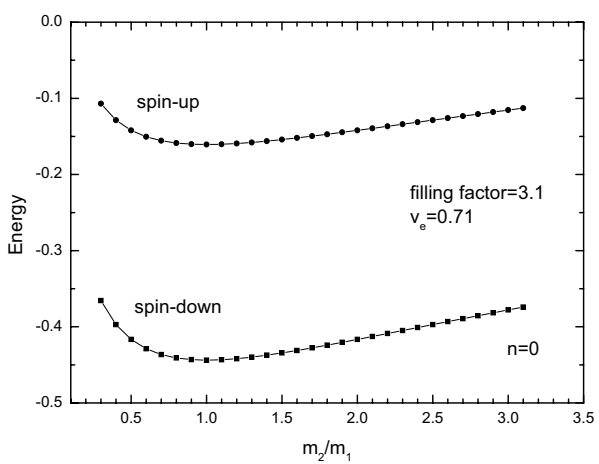

FIG. 2: (Color online) The energy of the lowest two Landau levels versus the effective mass ratio.

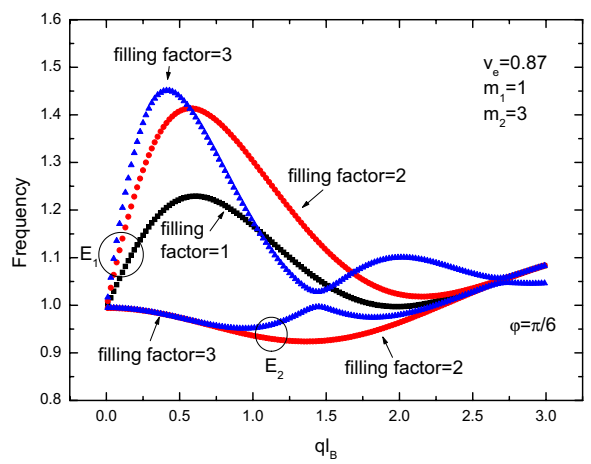

FIG. 3: (Color online) The frequency of magneto-plasmon modes versus the amplitude of the wave vector, for some integer filling factors.

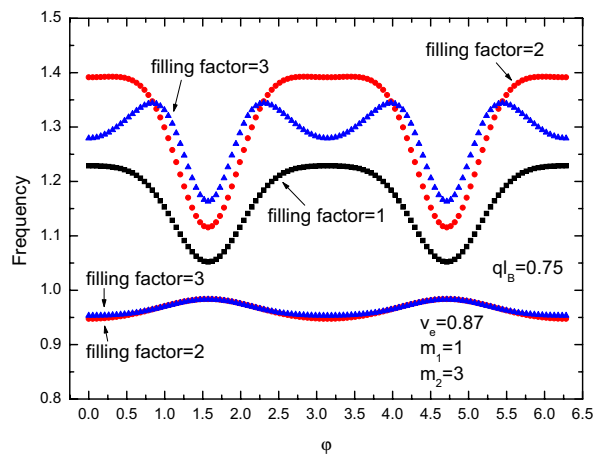

FIG. 4: (Color online) The frequency of magneto-plasmon modes versus the direction of the wave vector, for some integer filling factors. 


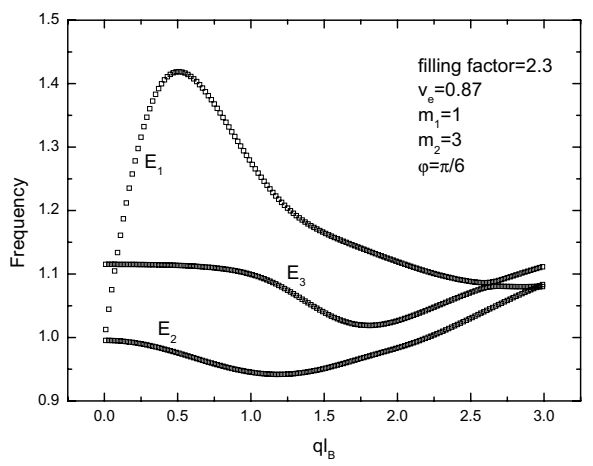

FIG. 5: (Color online) The frequency of magneto-plasmon modes versus the amplitude of the wave vector, for a noninteger filling factor.

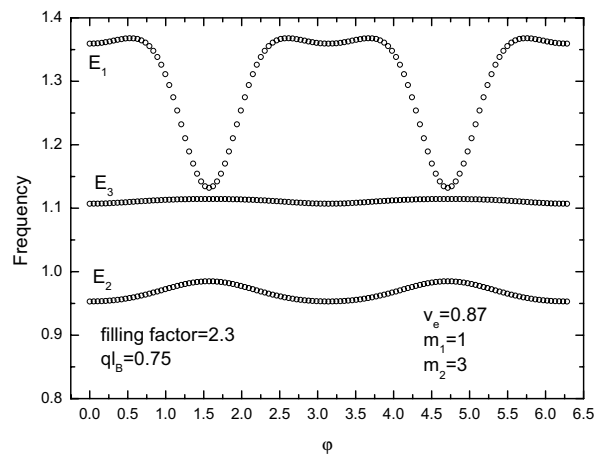

FIG. 6: (Color online) The frequency of magneto-plasmon modes versus the direction of the wave vector, for a noninteger filling factor. 